\title{
Overview of the POLAR mission
}

\author{
Jianchao Sun ${ }^{* a b}$ on behalf of the POLAR collaboration ${ }^{\dagger}$ \\ ${ }^{a}$ Key Laboratory of Particle Astrophysics, Institute of High Energy Physics, Chinese Academy of \\ Sciences, Beijing 100049, China \\ ${ }^{b}$ University of Chinese Academy of Sciences, Beijing 100049, China \\ E-mail: sunjc@ihep.ac.cn
}

The Gamma-ray Bursts Polarimeter POLAR mission is a dedicated experiment to measure and investigate the polarization details of the Gamma-ray Burst (GRB) prompt emissions with so far the highest precision under the close collaboration between Chinese and European scientists for more than ten years. POLAR has been successfully launched onboard the Chinese space laboratory Tiangong-2 on 15th September, 2016. The POLAR detector is optimized for the hard X-ray/Gamma-ray polarization measurement in 50-500 keV energy range with Compton scattering technology. Along with proper and detailed both ground and in-orbit calibrations and performance study, eventually the POLAR systematic effects are well controlled and relatively small after appropriate amendment. After launch, POLAR has detected 55 GRBs jointly with other instruments. Of these, detailed polarization measurements have been published recently for 5 of them. The analysis of the 5 GRBs shows that the average polarization degree is not as high as some GRB models predicted. A new finding of the evolution of the intrapulse polarization angle provides us with a new insight into the GRB physics. The latest results of POLAR aroused a big interest but also raised several critical scientific questions regarding GRB physics. Thus, the POLAR's successor mission which is POLAR-2 is proposed. Besides, POLAR also detected several Solar Flare events and observed and monitored bright pulsars during operation. POLAR is also able to roughly localize the direction for some bright GRBs. The design and implementation of the POLAR experiment, the performance and scientific observation results of POLAR will be addressed.

36th International Cosmic Ray Conference -ICRC2019-

July 24th - August 1st, 2019

Madison, WI, U.S.A.

\footnotetext{
* Speaker.

${ }^{\dagger}$ for collaboration list see PoS(ICRC2019)1177, and please visit http://polar.ihep.ac.cn/en/
} 


\section{Introduction}

It is commonly accepted that the polarization information during the prompt emission of Gamma-Ray Burst (GRB) is critical for unveiling the mystery of the radiation mechanisms of GRBs [1]. Therefore, more and more space instruments are trying to measure the polarization properties of GRBs [2]. However, most of the results suffer from systematic uncertainties [3] except for the GAP and COSI experiments $[4,5,6]$. Recently, the Gamma-Ray Bursts Polarimeter POLAR [7, 8] experiment has presented high precision polarization measurements of GRB prompt emissions and published the measurement details for five GRBs [9] which are so far the largest data sample for GRB prompt emission polarization measurement at a time. The joint polarization analysis for the five GRBs shows that the average polarization degree $(\sim 10 \%)$ is not as high as some GRB models predicted. A new finding of the evolution of the intrapulse polarization angle provides us with a new insight into the GRB physics. These new observations suggest that the rapid polarization angle change could be dominated by the internal evolution of the relativistic jets in which the intense bursts of Gamma-rays are produced.

The POLAR instrument was developed under the collaboration between China and Europe which lasted about ten years. The collaboration naturally continues for the in-orbit data analysis. POLAR was launched successfully onboard the Chinese space laboratory Tiangong-2 (TG-2) on 15th September, 2016. The POLAR mission finished in early April 2017, this was due to the failure of the high voltage power supply. This paper firstly reviews the design and development of the POLAR mission, then introduces the calibration and performance study activities on ground, finally presents POLAR's in-flight detection performance and scientific results, as well as expectations on future work with POLAR data and its successor which is POLAR-2 that has been officially selected as one of the international payloads onboard the future China space station (CSS).

\section{Instrument design and development}

The final product of POLAR mainly consists of two instruments: the polarimeter detector which is designated as OBOX and installed outside of TG-2 with zenith pointing always away from the Earth, while the other one is the electric cabinet which is named IBOX and installed inside of TG-2. OBOX is principally in charge of the scientific observation where the detection procedure happens and the generated data flows to IBOX through LVDS cable. IBOX receives the data from OBOX and transfers the data to TG-2, as well as receives commands from TG-2 and injects them into OBOX for implementation. TG-2 supplies $+100 \mathrm{~V}$ power supply to IBOX, and IBOX supplies $+28 \mathrm{~V}$ power supply to OBOX. The main detector body, OBOX, consists of 25 independent detector modular units (DMU), central trigger (CT), high-voltage power supply (HVPS), low-voltage power supply (LVPS), as well as mechanical structures and multi-layer insulator (MLI). Each DMU is composed of 64 low-Z material plastic scintillator (PS) bars, read out by a flat-panel multi-anode photomultiplier (MAPMT) and ASIC front-end electronics (FEE). The dimensions for each PS bar are $5.8 \times 5.8 \times 176 \mathrm{~mm}^{3}$ with a truncated cone shape at both ends of the bar which is used for reducing the crosstalk signals between each two PS bars. Each PS bar is a detection channel or pixel of the detector, therefore, POLAR has 1600 detection channels in total in a $40 \times 40$ array. 
More details about the POLAR instrument design can be found in [7]. Photos of OBOX and IBOX flight model (FM) are shown in Fig. 1.

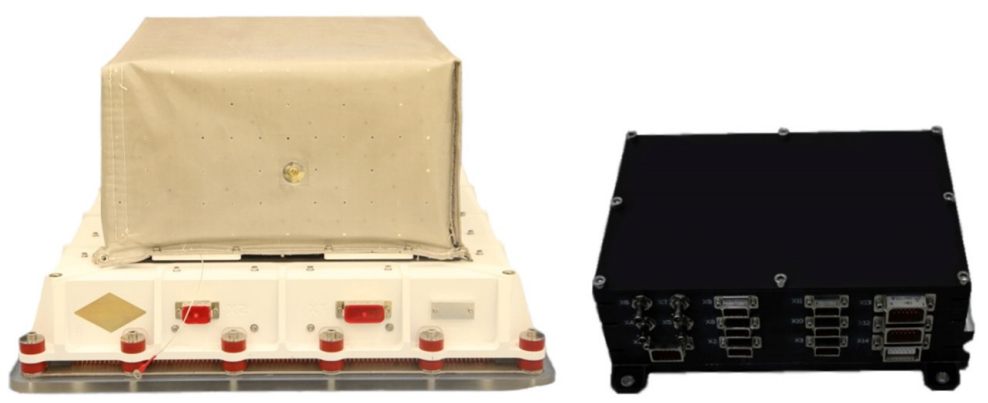

Figure 1: Photo of POLAR flight model. Left: OBOX. Right: IBOX.

\section{Ground calibration and performance study}

Validation of the detector working principle at the beginning of the design phase played an important role, as it determined if the scientific goal can be fulfilled and the design need to be updated. The main results can be found in [10,11]. Later on, all the compositions of the detector were developed in succession. One of the typical units is the DMU, thus its performance was also studied [12]. Finally, the integration of FM model was done and the calibration with $100 \%$ polarized hard X-rays at the European Synchrotron Radiation Facility (ESRF) was performed with lower beam energies of $110 \mathrm{keV}, 80 \mathrm{keV}$ and $60 \mathrm{keV}$ for both on-axis and off-axis tests, and the measured modulation factors agreed well with the Monte-Carlo simulation results. Lastly, the space environment tests should be done on each model of all the development phases, the only difference is that the level or conditions of the tests were different, e.g. qualification tests on qualification model $(\mathrm{QM})$ and acceptance tests on FM. Only after all the products passed all the space environment tests, they are space qualified. Some early studies about the qualification tests of POLAR can be found in [13].

\subsection{Performance at module level}

Assembling the POLAR DMU is a technically elaborate work which includes mainly four steps: 1) Screening of PS bars, MAPMT, FEE, baffles, etc.; 2) Matching of the PS bars and the baffles to make sure the PS bars exceeds the baffle holes $\sim 1 \mathrm{~mm}$; 3) Assembly of the PS target; 4) Coupling of the PS target with the FEE and MAPMT. The main procedures are shown in Fig. 2.

After assembly, all the DMUs should be tested to study their performance, and finally the selected DMUs for the FM model were determined based on the performance. Here the performance is the basic working performance of the DMU, such as the intrinsic noise and common noise, crosstalk, gain, non-uniformity of the gain among the 64 detection channels and low threshold. However, all these basic performances of the DMU will finally affect the polarization performance of POLAR, so they are also important to be analyzed. In [12] the basic analysis of DMU concluded that the average intrinsic noise of each DMU is typically below 20 ADC channel which means $\sim 1$ $\mathrm{keV}$; the non-uniformity of the gain for all the DMUs is below 3, typically $\sim 2$ and lowest 1.6; 


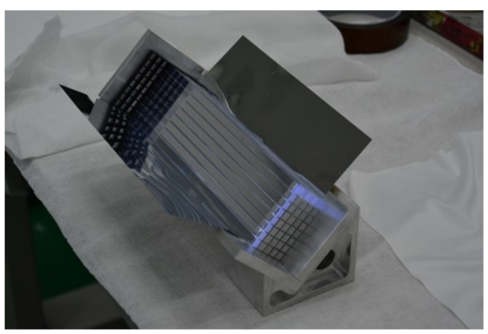

(a)

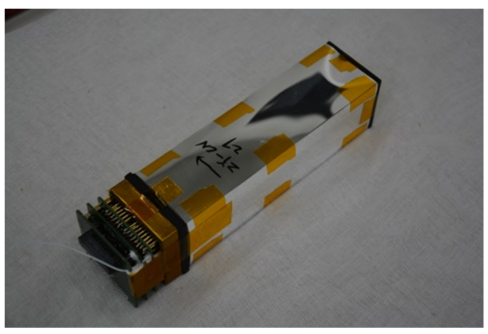

(c)

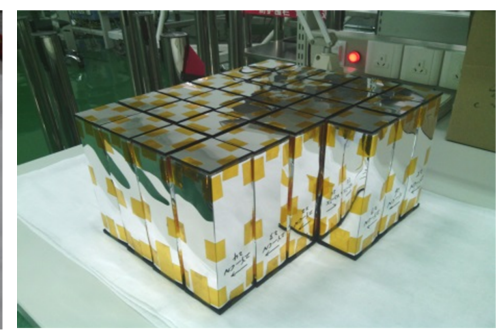

(b)

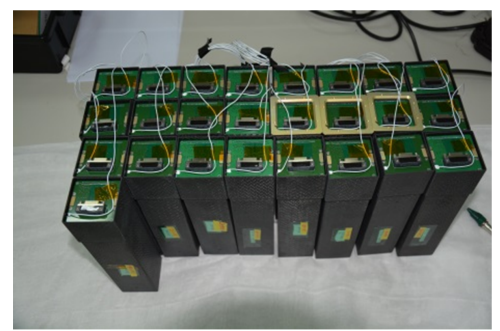

(d)

Figure 2: Assembly of POLAR detector modular unit (DMU). (a): Assembly of one plastic scintillator bars target. (b): Assembled plastic scintillator bars targets. (c): Naked POLAR DMU with PS target coupled to MAPMT and FEE. (d): POLAR DMU.

the average low threshold of each DMU is $\sim 15 \mathrm{keV}$. The tests were done at module level with a laboratory ${ }^{22} \mathrm{Na}$ radioactive source with the activity of $\sim 55 \mathrm{kBq}$.

\subsection{Calibration}

The calibration normally means the energy response production of the detector, including the redistribution matrix file (RMF) and ancillary response file (ARF). For POLAR, the most important calibration on ground is polarization measurement response which can not be easily tested in-orbit. This is mainly to verify the detector's polarization measurement precision and optimize the Monte-Carlo simulation software that is needed for GRB polarization measurement analysis.

There have been several polarization calibrations using synchrotron radiation facilities in the development phases for POLAR. Besides the ESRF beam test in 2009 with the original DMU, there was another polarization calibration with POLAR QM also at ESRF in 2012 and an important one with POLAR FM at ESRF again in 2015. A photo of POLAR OBOX at ESRF ID11 beamline during the polarization calibration in 2015 is shown in Fig. 3. During the ESRF beam test in 2015, POLAR FM was a full product including everything integrated, thus it produced a full polarization measurement response of the instrument. The later data analysis [14] showed that for beam energies of $110 \mathrm{keV}, 80 \mathrm{keV}$ and $60 \mathrm{keV}$, both on-axis and off-axis (for $30^{\circ}$ and $60^{\circ}$ off-axis) measured modulation factor agreed very well with the Monte-Carlo simulation results as for the Monte-Carlo simulation software it added the digitization procedure of the detector with the parameters from the basic performance calibrations. One important parameter which is the Birks's constant came from the measurement result of [15]. 
Other calibrations on ground of POLAR FM after integration such as the gain of the detector versus MAPMT high voltage with laboratory radioactive source can be found in [16] which will not be described in detail in this paper but also very important for the detector performance study and working parameter optimization.

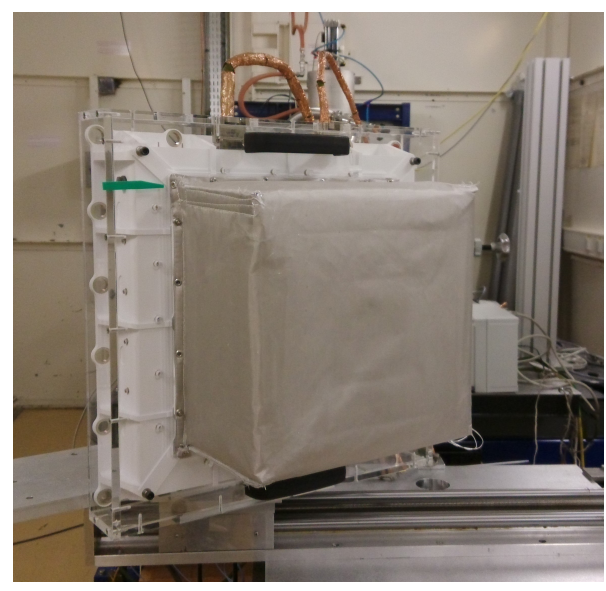

Figure 3: POLAR OBOX FM at ESRF for 100\% polarization beam test.

\section{In-orbit performance and observation results}

During its operation in orbit, POLAR performed well and was accurately calibrated in-orbit. In about six-months, 55 GRBs were detected and confirmed jointly with other GRB observation instruments, and detailed polarization measurement results with high precision have been reported for the prompt emissions of five GRBs recently [9]. This is so far the largest high-precision sample of polarization measurement of GRB prompt emissions. Besides, POLAR also detected the signals from Crab pulsar and solar flare (SFL), which inspires new expectations on further scientific outcome with POLAR data.

\subsection{In-orbit calibration}

There are four ${ }^{22} \mathrm{Na}$ radioactive sources installed inside POLAR OBOX FM which are used for POLAR in-orbit energy response calibrations and long-term performance monitoring. A photo of one of the four sources is shown in Fig. 4. The total radioactivity of the four sources is $\sim 350 \mathrm{~Bq}$. Besides the energy response, all the performances of the instrument have been studied thoroughly $[17,18]$, such as pedestal and noise, crosstalk, nonlinearity of gain, threshold, as well as the dependance of all the calibration parameters on the temperature. The final in-orbit calibration parameters were applied in the update of the digitization of the Monte-Carlo simulation software. In the same time, the typical calibration induced systematic error on polarization measurement was also analyzed which turned out to be less than $2 \%$. In addition, a preliminary study of the gain change using both in-orbit calibration data and ground data was performed [19]. The results show that after thermal and vibration factors correction, the in-orbit calibrated gain factor agreed well with the ground measurement results, which shows the stable and proper measurements of POLAR 
instrument both on ground and in-orbit, and proves our understanding of POLAR performance in-orbit is correct.

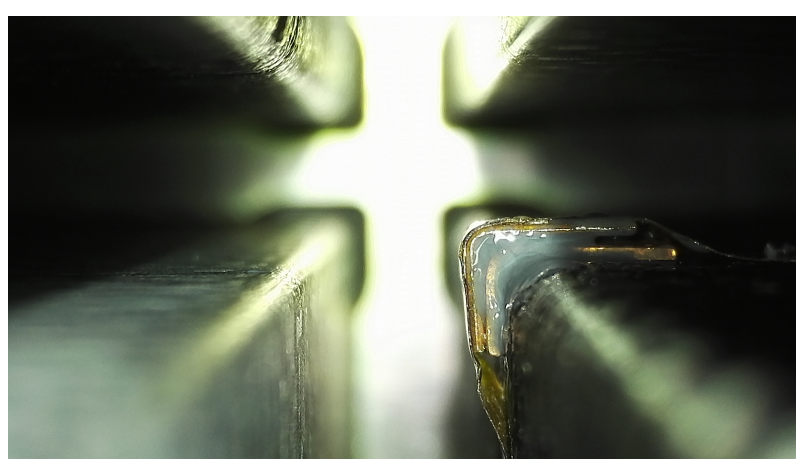

Figure 4: One of the in-orbit calibration ${ }^{22} \mathrm{Na}$ radioactive sources installed inside POLAR OBOX FM.

\subsection{GRB detection}

Considering the real in operation time and undiscovered GRBs, the GRB detection rate of 55 in $\sim 6$ months enables POLAR one of the most sensitive GRB detectors in operation. Among the 55 confirmed GRBs, joint observations include Fermi/GBM, Fermi/LAT, Swift/BAT, IPN, Konus-Wind, SPI-ACS, AstroSAT, et al. [20], and most of them are long GRBs. Some preliminary study of the joint data analysis between Fermi/GBM and POLAR has even been tried to find a solution for high priority POLAR data downloading and analysis using GBM's light curve and spectrum data [21].

\subsection{Polarization measurement of GRB prompt emission}

Analysis of the polarization measurement data is complicated and requires a lot of Monte-Carlo simulations. The measured modulation curve needs to be compared with the simulation results to find the most likely polarization possibility using either Least $\chi^{2}$ method or Bayesian method. The summary of detailed polarization measurement analysis results for five GRBs is shown in Table. 1. More details of the GRB polarization measurement analysis of POLAR can be found in $[9,22]$.

Table 1: Summary of the polarization measurement analysis results of the 5 GRBs and of 2 time bins of GRB 170114A (*in units of erg $/ \mathrm{cm}^{2}$ in $10-1000 \mathrm{keV}$ ) [9].

\begin{tabular}{|c|c|c|c|c|c|c|c|}
\hline GRB & T90 $(\mathrm{s})$ & Fluence* & PD & $\operatorname{Prob}(\mathrm{PD}<2 \%)$ & PD $_{\text {up }}(99 \%)$ & PA(deg.) & PA Change \\
\hline \hline $161218 \mathrm{~A}$ & 6.76 & $1.25 \times 10^{-5}$ & $9 \%$ & $9 \%$ & $45 \%$ & 40 & No \\
\hline $170101 \mathrm{~A}$ & 2.82 & $1.27 \times 10^{-5}$ & $8 \%$ & $13 \%$ & $31 \%$ & 164 & No \\
\hline $170127 \mathrm{C}$ & 0.21 & $7.4 \times 10^{-6}$ & $11 \%$ & $5.8 \%$ & $67 \%$ & 38 & Unknown \\
\hline $170206 \mathrm{~A}$ & 1.2 & $1.34 \times 10^{-5}$ & $10 \%$ & $12 \%$ & $31 \%$ & 106 & No \\
\hline $170114 \mathrm{~A}$ & 8.0 & $1.93 \times 10^{-5}$ & $4 \%$ & $14 \%$ & $28 \%$ & 164 & Yes \\
\hline $170114 \mathrm{Ap} 1$ & $\mathrm{~N} / \mathrm{A}$ & $\mathrm{N} / \mathrm{A}$ & $15 \%$ & $8 \%$ & $43 \%$ & 122 & N/A \\
\hline $170114 \mathrm{Ap} 2$ & $\mathrm{~N} / \mathrm{A}$ & $\mathrm{N} / \mathrm{A}$ & $41 \%$ & $0.49 \%$ & $74 \%$ & 17 & $\mathrm{~N} / \mathrm{A}$ \\
\hline
\end{tabular}




\subsection{Expectation on future work}

For the polarization analysis on the other GRBs than the five ones that have been published, it is more complicate as either the incident angles are larger or the brightness of the GRBs is relatively weak. However, the method used in [22] can be applied. The Crab pulsar navigation method test with POLAR data has been preliminarily studied and proved to be quite promising [23], and more work can be done on this. Besides, the potential for polarization analysis of the Crab pulsar with POLAR data is also expected to be performed after finalizing current studies on Crab spectral measurements using the POLAR data. Analysis show that POLAR is able to localize bright GRBs with expected precisions, more analysis work is undergoing now. The in-orbit background of POLAR is complicate even though it does not affect the final polarization analysis. However, to acquire a much better minimum detectable polarization (MDP) in the future for GRB polarimetry at lower energies, e.g. for POLAR-2, restraining as much background as possible is needed. Thus it is worth to study this in detail. POLAR also detected several SFL events in-orbit which are potentially can be used for polarization analysis. All in all, the POLAR data is valuable and many analysis work can be done with the data in the next steps.

\section{Conclusions and outlook}

The design and development of POLAR instrument lasted $\sim 10$ years. Both ground and in-orbit performance of the polarimeter presented very good polarization measurement abilities and precision. Recently, detailed polarization measurement results of five GRBs by POLAR have been published which is so far the largest measurement sample for GRB polarizations during its prompt emissions. Even though POLAR has achieved its scientific goal and extended its scientific detection abilities during flight, it was really unfortunate that POLAR broke down due to the HVPS failure. However, there are still many expectations on more scientific outcome by analyzing POLAR's data.

Thanks to POLAR's new findings, scientists from China, Switzerland, Germany, Poland have formed an enlarged collaboration team to develop a much more powerful polarimeter which is POLAR-2 [24], as the successor of the POLAR experiment. POLAR-2 is intended to be onboard China Space Station to address the new scientific questions raised by POLAR, ultimately helping to understand the nature of GRBs.

\section{References}

[1] K. Toma, et al. Statistical properties of gamma-ray burst polarization. Astrophys. J., 2009, 698: 1042.

[2] S. Covino, D. Götz. Polarization of prompt and aferglow emission of gamma-ray bursts. Astron. Astrophys. Trans., 2016, 29: 205.

[3] M. McConnel. High energy polarimetry of prompt GRB emission. New Astr. Rev., 2017, 76: 1.

[4] A. W. Lowell, et al. Polarimetric analysis of the long duration gamma-ray burst GRB 160530A with the balloon borne Compton Spectrometer and Imager. Astrophys. J. Lett., 2017, 848: 119.

[5] D. Yonetoku, et al. Magnetic structures in gamma-ray burst jets probed by gamma-ray polarization. Astrophys. J. Lett., 2012, 758: L1. 
[6] D. Yonetoku, et al. Detection of gamma-ray polarization in prompt emission of GRB 100826A. Astrophys. J. Lett., 2011, 743: L30.

[7] N. Produit, et al. Design and construction of the POLAR detector. Nucl. Instrum. Methods Phys. Res. A, 2018, 877: 259.

[8] N. Produit, et al. POLAR, a compact detector for gamma-ray bursts photon polarization measurements. Nucl. Instrum. Methods Phys. Res. A, 2005, 550: 616.

[9] S. N. Zhang, M. Kole, et al. Detailed polarization measurements of the prompt emission of five gamma-ray bursts. Nature Astronomy, 2019, 3: 258.

[10] E. Suarez-Garcia. Ph.D thesis: "X-Ray Polarization: RHESSI Results and POLAR Prospects", Switzerland: University of Geneva (2010).

[11] S. Orsi, et al. Response of the Compton polarimeter POLAR to polarized hard X-rays. Nucl. Instrum. Methods Phys. Res. A, 2011, 648: 139.

[12] J. C. Sun, et al. Performance study of the gamma-ray bursts polarimeter POLAR. Proc. of SPIE Vol. 9905, 2016.

[13] J. C. Sun, et al. Qualification Tests of the Space-Based POLAR X-Ray Polarimeter. Proc. of ICRC, 1351, 2011.

[14] M. Kole, et al. Instrument performance and simulation verification of the POLAR detector. Nucl. Instrum. Methods Phys. Res. A, 2017, 872: 28.

[15] X. F. Zhang, et al. Study of non-linear energy response of POLAR plastic scintillators to electrons. Nucl. Instrum. Methods Phys. Res. A, 2015, 797: 94.

[16] X. F. Zhang, et al. Optimization of the gain factors and parameter settings for the new gamma-ray burst polarimeter, POLAR. Nucl. Instrum. Methods Phys. Res. A, 2018, 879: 47.

[17] Z. H. Li, et al. In-orbit instrument performance study and calibration for POLAR polarization measurements. Nucl. Instrum. Methods Phys. Res. A, 2018, 900:8.

[18] H. L. Xiao, et al. In-flight energy calibration of the space-borne Compton polarimeter POLAR. Astroparticle Physics, 2018, 103: 74.

[19] J. C. Sun, et al. Preliminary Study of the In-orbit Gain Performance of the Gamma-Ray Bursts Polarimeter - POLAR (in Chinese). Proc. of 31st national symposium on space exploration of China, 2018.

[20] S. L. Xiong, et al. Overview of the GRB observation by POLAR. Proc. of ICRC, 640, 2017.

[21] Y. Zhao, et al. A low-latency pipeline for GRB light curve and spectrum using Fermi/GBM near real-time data. RAA, 2018, 18(5): 57.

[22] J. M. Burgess, et al. Time-Resolved GRB Polarization with POLAR and GRB Simultaneous spectral and polarization analysis with synchrotron emission. A \& A, 2019, 627, arXiv:1901.04719.

[23] S. J. Zheng, et al. Test of pulsar navigation with POLAR on TG-2 spacelab (in Chinese). SCIENTIA SINICA Physica, Mechanica \& Astronomica, 2017, 47(9): 099505.

[24] M. Kole. POLAR-2: The First Large Scale Gamma-Ray Polarimeter. Proc. of ICRC, 2019. 